\title{
A New Descent Algebra for Weyl Groups of Type $A_{n}$
}

\author{
Tülay YAĞMUR ${ }^{1}$, Himmet $\mathrm{CAN}^{2}$ \\ 1. Vocational School of Social Sciences, Aksaray University, 68100 Aksaray, Turkey. \\ 2. Department of Mathematics, Erciyes University, 38039 Kayseri, Turkey.
}

Received: April 22, 2016 / Accepted: May 18, 2016 / Published: June 25, 2016.

\begin{abstract}
In this paper we define an equivalence relation on the set of all $x_{J}$ in order to form a basis for a new descent algebra of Weyl groups of type $A_{n}$. By means of this, we construct a new commutative and semi-simple descent algebra for Weyl groups of type $A_{n}$ generated by equivalence classes arising from this equivalence relation.
\end{abstract}

Keywords: Weyl groups; type $A_{n}$; descent algebra.

\section{Introduction}

The main objective of this paper is to construct a new descent algebra for Weyl groups of type $A_{n}$ by using Solomon's basic concepts. Moreover, the basis of this new algebra consists of equivalence classes arising from the equivalence relation on the set $x_{J}$, which is going to be defined in this section. This new descent algebra will be denoted by $\sum_{W}\left(A_{n}\right)$.

In the next section, we shall determine two basic properties of this new descent algebra. That is to say, by contrast with the Solomon's descent algebra, we shall show that this new algebra is commutative and also semi-simple. Furthermore, we shall show that there is an isomorphism between the new descent algebra $\sum_{W}\left(A_{n}\right)$ and the parabolic Burnside ring of associated Weyl group. Note that, there is a homomorphism between the Solomon algebra $\sum(W)$ and the parabolic Burnside ring $P B(W)$ [3]. In addition, we shall show that there is an isomorphism between the new descent algebra $\sum_{W}\left(A_{n}\right)$ and the $Z$-module of class functions $G\left(W\left(A_{n}\right)\right)$ generated by permutation character $\chi_{J}$.

Now the notation, which is fairly standard and

Corresponding author: Tülay YAĞMUR, Vocational School of Social Sciences, Aksaray University, 68100 Aksaray, Turkey. E-mail: tulayyagmurr@gmail.com. follows that given in Carter [1], and Solomon [2] or Bergeron et al. [3], is first summarized.

Let $\Phi$ be a root system with fundamental system $\Pi$ and corresponding positive system $\Phi^{+}$. If $r \in \Phi$, let $w_{r}$ denote the reflection corresponding to $r$. Besides, let $W=W(\Phi)=W(\Pi)$ be the Weyl group of root system $\Phi$, that is, the group generated by the $w_{r}, r \in \Phi$.

Let $e_{1}, e_{2}, \ldots, e_{n+1}$ be an orthonormal basis of a Euclidean space of dimension $n+1$. Then the fundamental system and root system of type $A_{n}$ are given by

$$
\begin{gathered}
\Pi=\left\{e_{1}-e_{2}, e_{2}-e_{3}, \ldots, e_{n-1}-e_{n}, e_{n}-e_{n+1}\right\}, \\
\Phi=\left\{e_{i}-e_{j} \mid i \neq j, \quad i, j=1, \ldots, n+1\right\},
\end{gathered}
$$

respectively.

Let $\Pi$ be a fundamental system in root system $\Phi$ of type $A_{n}$ and $\Phi^{+}$be the corresponding positive system. Then $W\left(A_{n}\right)$ is called the Weyl group of type $A_{n}$ generated by the reflections $w_{r}$ for all $r \in \Phi$.

The descent algebra $\sum(W)$ of a finite Coxeter group $W$ had been discovered by Solomon in 1976 . After this seminal paper [2], there have been huge developments in the area of descent algebras of Coxeter groups over the last four decades. One can see 
for these developments, for example [3], [4] and [6].

Let $W_{J}$ be the subgroup of $W$, where $J$ is any subset of $\Pi . W_{J}$ is called a standard parabolic subgroup of $W$. Let $X_{J}$ be the set of representatives of the cosets of $w W_{J}$ in $W$. Furthermore, $X_{J}^{-1}=\left\{w^{-1} \mid w \in X_{J}\right\} \quad$ is a set of representatives for the right cosets of $W_{J}$ in $W$. Then $X_{J K}=X_{J}^{-1} \bigcap X_{K}$ is a set of distinguished double coset representatives of $W_{J} w W_{K}$ in $W$.

The following remarkable theorem has been proved by Solomon [2].

Theorem 1.1 For every subset $J$ of $\Pi$, let $x_{J}=\sum_{d \in X_{J}} d$. Then

$$
x_{J} x_{K}=\sum_{L \subseteq K} a_{J K L} x_{L},
$$

where $a_{J K L}$ is the number of elements $w \in X_{J K}$ such that $w^{-1}(J) \cap K=L$.

It follows that the set of all $x_{J}$ form a basis for a noncommutative algebra $\sum(W)$ over the field of rationals. This algebra is called the descent algebra (or Solomon algebra) of Coxeter groups $W$ [3].

The descent algebra has been reconstructed by Bergeron et al. in 1992 [3]. In their paper, they have given a relatively elegant construction for the Solomon's descent algebra. Additionally, they have introduced the parabolic Burnside ring of the associated Coxeter group. Moreover, there is a natural homomorphism from the descent algebra $\sum(W)$ into the parabolic Burnside ring of the associated Coxeter group.

Clearly, since the Weyl groups are also finite Coxeter groups then the construction of their descent algebra is valid for Weyl groups.

In other respects, the descent algebra associated with the Weyl groups of type $A_{n}$ has been intensively studied. For example, Garsia and Reutenauer [7] have given an analysis of the descent algebra of the symmetric group. Besides, Atkinson [5] has determined the Loewy length of the descent algebra of the symmetric group.

Now, for our purpose, we need to define an equivalence relation on the set of all $x_{J}$, where $J \subseteq \Pi$. By this way, we can obtain the equivalence classes in order to form the basis for a new descent algebra. Because of this, we now give the following results.

Definition 1.2 For $J, K \subseteq \Pi$, we write $J \sim K$ whenever $w(J)=K$ for some $w \in W$, that is, $J$ and $K$ are conjugate [3].

This concept is used by Solomon [2] for any Weyl groups as follows.

Lemma 1.3 If $J, K \subseteq \Pi$ then $J \sim K$ if and only if $W_{J}$ and $W_{K}$ are conjugate, that is, $W_{K}=w W_{J} w^{-1}$ for some $w \in W$.

Now, we define a relation on the set of all $x_{J}$ in order to form a basis for a new descent algebra of Weyl groups of type $A_{n}$ as follows:

$$
x_{J} \sim x_{K} \Leftrightarrow J \sim K \Leftrightarrow K=w(J), \quad \text { for } \quad w \in W \text {. }
$$

It is clear that it is an equivalence relation. By means of this equivalence relation, for $J \subseteq \Pi$, the equivalence class which contains $x_{J}$ is

$$
\begin{aligned}
{\left[x_{J}\right] } & =\left\{x_{K} \mid x_{K} \sim x_{J}, K \subseteq \Pi\right\} \\
& =\left\{x_{K} \mid K \sim J\right\} \\
& =\left\{x_{w(J)} \mid w(J) \subseteq \Pi, w \in W\right\} .
\end{aligned}
$$

The main result of our paper is the following:

Theorem A. Let $J \subseteq \Pi$, then

$$
\sum_{W}\left(A_{n}\right)=\operatorname{Sp}\left\{\left[x_{J}\right] \mid J \subseteq \Pi\right\}
$$

is a new descent algebra of Weyl groups of type $A_{n}$.

\section{Proof of Theorem A}

In this section, we shall obtain some significant results to prove Theorem A, and so we shall complete the construction. Furthermore, we shall obtain substantial results stating in Section 1.

In that case, recall that the set $\left\{x_{J} \mid J \subseteq \Pi\right\}$ is a basis for the Solomon's descent algebra $[2,3]$. Then it 
is obvious that the set $\left\{\left[x_{J}\right] \mid J \subseteq \Pi\right\}$ is linearly independence, and also a basis for $\sum_{W}\left(A_{n}\right)$. Therefore $\sum_{W}\left(A_{n}\right)$ is a $Q$-space, which is generated by all different equivalence classes $\left[x_{J}\right]$, where $J \subseteq \Pi$.

Besides, this $Q$-space $\sum_{W}\left(A_{n}\right)$ must have a ring structure for our purpose. Because of this, we now define a ring multiplication as follows:

Let $J, K \subseteq \Pi$, then

$$
\left[x_{J}\right]\left[x_{K}\right]=\left[x_{J} x_{K}\right]=\sum_{L \subseteq K} a_{J K L}\left[x_{L}\right]
$$

where the $a_{J K L}$ 's are defined as Section 1 .

The following theorem shows that this multiplication is well-defined.

Theorem 2.1 If $J, J^{\prime}, K, K^{\prime} \subseteq \Pi, J \sim J^{\prime}$ and $K \sim K$ then

$$
\left[x_{J}\right]\left[x_{K}\right]=\left[x_{J}\right]\left[x_{K}{ }^{\prime}\right] .
$$

Now, for the proof of this theorem, we need to state the following crucial lemmas.

Lemma 2.2 If $J, J^{\prime}, K, K^{\prime} \subseteq \Pi, J \sim J^{\prime}$ and $K \sim K$ then

$$
\left|W_{J}\right| W / W_{K}|=| W_{J},\left|W / W_{K},\right| .
$$

Proof. If $J \sim J^{\prime}$ and $K \sim K^{\prime}$ then there exist at least $w \in W$ and $\sigma \in W$ such that $J^{\prime}=w(J)$ and $K^{\prime}=\sigma(K)$, respectively. Furthermore, by Lemma 1.3 , we can write $W_{J^{\prime}}=w W_{J} w^{-1}$ and $W_{K}=\sigma W_{J} \sigma^{-1}$.

Now we define a map as follows to achieve our goal:

$$
\begin{gathered}
\Psi: W_{J}\left|W / W_{K} \rightarrow W_{J},\right| W / W_{K^{\prime}} ; \\
\Psi\left(W_{J} z W_{K}\right)=W_{J}, w z \sigma^{-1} W_{K}^{\prime}
\end{gathered}
$$

for $z \in W$.

The map $\Psi$ is well-defined because, if

$$
W_{J} z_{1} W_{K}=W_{J} z_{2} W_{K}
$$

then we obtain

$$
\begin{aligned}
\Psi\left(W_{J} z_{1} W_{K}\right) & =W_{J}, w z_{1} \sigma^{-1} W_{K^{\prime}} \\
& =w W_{J} z_{1} W_{K} \sigma^{-1} \\
& =w W_{J} z_{2} W_{K} \sigma^{-1} \\
& =\Psi\left(W_{J} z_{2} W_{K}\right) .
\end{aligned}
$$

Obviously, if

$$
\Psi\left(W_{J} z_{1} W_{K}\right)=\Psi\left(W_{J} z_{2} W_{K}\right)
$$

then we have

$$
W_{J} z_{1} W_{K}=W_{J} z_{2} W_{K}
$$

Also, if we consider $W_{J} v W_{K}, \in W_{J} \mid W / W_{K}$, then there exist $W_{J} w^{-1} v \sigma W_{K} \in W_{J} \mid W / W_{K}$ such that

$$
\Psi\left(W_{J} w^{-1} v \sigma W_{K}\right)=W_{J} v W_{K} \cdot .
$$

So, this means that $\Psi$ is a bijective. Thus, the sets $W_{J} \mid W / W_{K}$ and $W_{J} \mid W / W_{K^{\prime}}$ have the same cardinality. This completes the proof.

This lemma immediately implies the following corollary.

Corollary 2.3 For $J, J^{\prime}, K, K^{\prime} \subseteq \Pi$, if $J \sim J^{\prime}$ and $K \sim K$ then

$$
\left|X_{J K}\right|=\left|X_{J_{K}^{\prime}}\right| \text {. }
$$

Also by Lemma 2.2 and Corollary 2.3, we can see that there is one-to-one correspondence between the elements of $X_{J K}$ and $X_{J^{\prime} K^{\prime}}$. Because of this reason, we can obtain

$$
W_{J} f W_{K}^{\prime}=W_{J} w d \sigma^{-1} W_{K}=w W_{J} d W_{K} \sigma^{-1} .
$$

Accordingly, we can say that there exist $w_{J} \in W_{J}$ and $w_{K} \in W_{K}$ such that $f=w w_{J} d w_{K} \sigma^{-1}$.

The following theorem is very essential for our 
costruction.

Theorem 2.4 Let $J, K \subseteq \Pi$ and $w \in X_{J K}$. Then

$$
W_{J} \bigcap w W_{K} w^{-1}=W_{L}
$$

where $L=J \cap w(K)$ [3].

Now, we can give the following lemma.

Lemma 2.5 For $J, J, K, K \subseteq \Pi, J \sim J^{\prime}$ and $K \sim K^{\prime}$, if $d \in X_{J K}$ and $f \in X_{J_{K}^{\prime}}$, then

$$
\left[x_{f^{-1}\left(J^{\prime}\right) \cap K^{\prime}}\right]=\left[x_{d^{-1}(J) \cap K}\right] .
$$

Proof. By Lemma 1.3, if $J \sim J$ and $K \sim K$ then there exist at least $w \in W$ and $\sigma \in W$ such that $W_{J}=w W_{J} w^{-1} \quad$ and $\quad W_{K}=\sigma W_{J} \sigma^{-1}$, respectively. And also by Theorem 2.4, we know that the group $W_{f^{-1}\left(J^{\prime}\right) \cap K^{\prime}}$ is equal to $f^{-1} W_{J^{\prime}} f \bigcap W_{K^{\prime}}$. Then

$$
\begin{aligned}
& W_{f^{-1}\left(J^{\prime}\right) \cap K^{\prime}} \\
& =f^{-1} W_{J} f \bigcap W_{K^{\prime}} \\
& =\sigma_{w_{K}}{ }^{-1} d^{-1} w_{J}^{-1} w^{-1} w W_{J} w^{-1} w w_{J} d w_{K} \sigma^{-1} \bigcap \sigma W_{K} \sigma^{-1} \\
& =\sigma w_{K}{ }^{-1}\left(d^{-1} W_{J} d \bigcap W_{K}\right) w_{K} \sigma^{-1} \\
& =\sigma w_{K}{ }^{-1} W_{d^{-1}(J) \cap K}\left(\sigma w_{K}{ }^{-1}\right)^{-1} .
\end{aligned}
$$

Note that, since $\sigma w_{K}^{-1} \in W$ then we obtain $W_{f^{-1}\left(J^{\prime}\right) \cap K^{\prime}} \sim W_{d^{-1}(J) \cap K^{\prime}}$. This fact help us to obtain $f^{-1}\left(J^{\prime}\right) \cap K^{\prime} \sim d^{-1}(J) \cap K$, so this means that

$$
\left[x_{f^{-1}\left(J^{\prime}\right) \cap K^{\prime}}\right]=\left[x_{d^{-1}(J) \cap K}\right] .
$$

This completes the proof.

We now have all the ingredients to give the proof of Theorem 2.1.

Proof of Theorem 2.1.

Let $J, J^{\prime}, K, K^{\prime} \subseteq \Pi, J \sim J^{\prime}$ and $K \sim K^{\prime}$.
Then

$$
\begin{aligned}
& {\left[x_{J},\right]\left[x_{K}{ }^{\prime}\right]=\left[x_{J} x_{K^{\prime}} x^{\prime}\right]} \\
& =\sum_{f \in X_{J^{\prime}}}\left[x_{f^{-1}\left(J^{\prime}\right) \cap K^{\prime}}\right] \\
& =\sum_{d \in X_{J K}}\left[x_{d^{-1}(J) \cap K}\right] \\
& =\left[x_{J}\right]\left[x_{K}\right],
\end{aligned}
$$

and Theorem 2.1 is proved.

Hence, the $Q$-space $\sum_{W}\left(A_{n}\right)=\operatorname{Sp}\left\{\left[x_{J}\right] \mid J \subseteq \Pi\right\}$ is a ring with identity $\left[x_{\Pi}\right]$. Obviously, this means that $\sum_{W}\left(A_{n}\right)$ is an algebra over the field of rationals. Thus, the construction of the algebraic structure has been completed and also by means of this we complete the proof of Theorem A. To sum up, $\sum_{W}\left(A_{n}\right)$ is a new descent algebra of Weyl groups of type $A_{n}$.

Now, we want to determine two basic properties of this new descent algebra $\sum_{W}\left(A_{n}\right)$, that is, this new descent algebra is commutative and also semi-simple.

By $[2,3,4]$, it is known that the radical of the descent algebra of Weyl groups is spanned by all differences $x_{J}-x_{K}$, where $J$ and $K$ are conjugate subsets of $\Pi$, that is to say,

$$
\operatorname{Rad} \sum(W)=\operatorname{Sp}\left\{x_{J}-x_{K} \mid J \sim K, \quad J, K \subseteq \Pi\right\} .
$$

Thus, by using this definition, we can obtain the radical of the new descent algebra of Weyl groups of type $A_{n}$ as follows:

$$
\operatorname{Rad} \sum_{W}\left(A_{n}\right)=\{0\} .
$$

Clearly, the next corollary is the consequence of this result.

Corollary 2.6 The new descent algebra $\sum_{W}\left(A_{n}\right)$ of Weyl groups of type $A_{n}$ is semi-simple.

The other property of this algebra is given as follows. 
Proposition 2.7 The new descent algebra $\sum_{W}\left(A_{n}\right)$ of Weyl groups of type $A_{n}$ is commutative.

Proof. Let $J, K \subseteq \Pi$. Then

$$
\begin{gathered}
{\left[x_{J}\right]\left[x_{K}\right]=\sum_{d \in X_{J K}}\left[x_{d^{-1}(J) \cap K}\right]} \\
\quad=\sum_{d^{-1} \in X_{K J}}\left[x_{d(K) \cap J}\right] \\
\quad=\sum_{f \in X_{K J}}\left[x_{f^{-1}(K) \cap J}\right] \\
=\left[x_{K}\right]\left[x_{J}\right] .
\end{gathered}
$$

So, Proposition 2.7 is proved.

Remark 2.8 We have seen that the new descent algebra $\sum_{W}\left(A_{n}\right)$ of Weyl groups of type $A_{n}$ generated by equivalence classes is different from the Solomon algebra $\sum(W)$ of Weyl groups of type $A_{n}$, since Solomon algebra is non-commutative and not semi-simple.

The following example illustrates Theorem A.

Example 2.9 Let us give the multiplication table for the new descent algebra $\sum_{W}\left(A_{3}\right)$, where the fundamental system of type $A_{3}$ is

$$
\Pi=\left\{e_{1}-e_{2}, e_{2}-e_{3}, e_{3}-e_{4}\right\} .
$$

By Theorem A, we know that the new descent algebra of Weyl group type $A_{3}$ is generated by the equivalence classes arising from the equivalence relation (1) defined in Section 1. Thus, by using this equivalence relation and also with keeping in mind the distinguished basis of the Solomon's descent algebra of Weyl group of type $A_{3}$, which is given by the sums of the distinguished coset representatives of parabolic subgroups of $W\left(A_{3}\right)$, then we can obtain the generators of this new descent algebra as follows:

$$
\left[x_{J}\right] \text {, where } J=\Pi \text {, }
$$

$\left[x_{K}\right]=\left[x_{L}\right]$, where $K=\left\{e_{1}-e_{2}, e_{2}-e_{3}\right\}$ or

$$
L=\left\{e_{2}-e_{3}, e_{3}-e_{4}\right\} \text {, }
$$

$$
\begin{aligned}
& {\left[x_{M}\right] \text {, where } M=\left\{e_{1}-e_{2}, e_{3}-e_{4}\right\} \text {, }} \\
& {\left[x_{N}\right]=\left[x_{P}\right]=\left[x_{Q}\right] \text {, }} \\
& \text { where } N=\left\{e_{1}-e_{2}\right\} \\
& \text { or } \\
& P=\left\{e_{2}-e_{3}\right\} \quad \text { or } \\
& Q=\left\{e_{3}-e_{4}\right\} \\
& {\left[x_{R}\right] \text {, where } R=\varnothing \text {. }}
\end{aligned}
$$

If we denote these elements by $x_{\lambda_{1}}, x_{\lambda_{2}}, x_{\lambda_{3}}$, $x_{\lambda_{4}}$ and $x_{\lambda_{5}}$, respectively, where $\lambda_{1}=J, \lambda_{2}=K$ or $\mathrm{L}, \lambda_{3}=M, \lambda_{4}=N$ or $P$ or $Q, \lambda_{5}=R$, then we get Table 1 as follows by using ring multiplication operation (2).

This example shows that the new commutative descent algebra of Weyl group of type $A_{3}$ has less elements than the Solomon's algebra associated with $W\left(A_{3}\right)$.

In other respects, the descent algebra is closely related to the subring of the Burnside ring $B(W)$. This ring is called the parabolic Burnside ring and denoted by $P B(W)$. Furthermore, the parabolic Burnside ring is spanned by the permutation representations $W / W_{J}$, where the $W_{J}$ are the parabolic subgroups of $W$. And also, the multiplication for $W / W_{J}$ and $W / W_{K}$ is defined by

$$
W / W_{J} \times W / W_{K}=\sum_{L \subseteq K} a_{J K L} W / W_{L}
$$

where the $a_{J K L}$ 's are defined as Section 1 [3].

Now we can state the following proposition.

Proposition 2.10 Let $\varphi: \sum_{W}\left(A_{n}\right) \rightarrow P B\left(W\left(A_{n}\right)\right)$ be the map given by $\varphi\left(\left[x_{J}\right]\right)=W / W_{J}$. This is an isomorphism.

Proof. This mapping is well-defined. In fact, if we take $\left[x_{J}\right]=\left[x_{K}\right]$ for $J, K \subseteq \Pi$ then we can obtain $W / W_{J} \cong W / W_{K}$ because of $J \sim K$ (see [3]). So, this implies that $\varphi\left(\left[x_{J}\right]\right)=\varphi\left(\left[x_{K}\right]\right)$. 
Table 1 The ring multiplication table of $\sum_{W}\left(A_{3}\right)$.

\begin{tabular}{|l|l|l|l|l|l|}
\hline & $x_{\lambda_{1}}$ & $x_{\lambda_{2}}$ & $x_{\lambda_{3}}$ & $x_{\lambda_{4}}$ & $x_{\lambda_{5}}$ \\
\hline$x_{\lambda_{1}}$ & $x_{\lambda_{1}}$ & $x_{\lambda_{2}}$ & $x_{\lambda_{3}}$ & $x_{\lambda_{4}}$ & $x_{\lambda_{5}}$ \\
\hline$x_{\lambda_{2}}$ & $x_{\lambda_{2}}$ & $x_{\lambda_{2}}+x_{\lambda_{4}}$ & $2 x_{\lambda_{4}}$ & $2 x_{\lambda_{4}}+x_{\lambda_{5}}$ & $4 x_{\lambda_{5}}$ \\
\hline$x_{\lambda_{3}}$ & $x_{\lambda_{3}}$ & $2 x_{\lambda_{4}}$ & $2 x_{\lambda_{3}}+x_{\lambda_{5}}$ & $2 x_{\lambda_{4}}+2 x_{\lambda_{5}}$ & $6 x_{\lambda_{5}}$ \\
\hline$x_{\lambda_{4}}$ & $x_{\lambda_{4}}$ & $2 x_{\lambda_{4}}+x_{\lambda_{5}}$ & $2 x_{\lambda_{4}}+2 x_{\lambda_{5}}$ & $2 x_{\lambda_{4}}+5 x_{\lambda_{5}}$ & $12 x_{\lambda_{5}}$ \\
\hline$x_{\lambda_{5}}$ & $x_{\lambda_{5}}$ & $4 x_{\lambda_{5}}$ & $6 x_{\lambda_{5}}$ & $12 x_{\lambda_{5}}$ & $24 x_{\lambda_{5}}$ \\
\hline
\end{tabular}

Additionally,one can easily verify that $\varphi$ is a bijective. To show that $\varphi$ is an isomorphism, it is enough to show that $\varphi\left(\left[x_{J}\right]\left[x_{K}\right]\right)=\varphi\left(\left[x_{J}\right]\right) \varphi\left(\left[x_{K}\right]\right)$. By using the equations (2) and (3), we obtain

$$
\begin{aligned}
\varphi\left(\left[x_{J}\right]\left[x_{K}\right]\right) & =\varphi\left(\left[x_{J} x_{K}\right]\right) \\
& =\varphi\left(\sum_{L \subseteq K} a_{J K L}\left[x_{L}\right]\right) \\
& =\sum_{L \subseteq K} a_{J K L} \varphi\left(\left[x_{L}\right]\right) \\
& =\sum_{L \subseteq K} a_{J K L} W / W_{L} \\
& =W / W_{J} \times W / W_{K} \\
& =\varphi\left(\left[x_{J}\right]\right) \varphi\left(\left[x_{K}\right]\right),
\end{aligned}
$$

and thus, Proposition 2.10 is proved.

Let $\chi_{J}$ be the permutation character of $W\left(A_{n}\right)$ acting on the left cosets of $W_{J}$ and let $G(W)$ be the $Z$-module generated by all $\chi_{J}[3,4]$. The following proposition was proved in [3].

Proposition 2.11 The assignment $W / W_{J} \mapsto \chi_{J}$ defines an isomorphism $\Theta$ from $P B(W)$ to the $G(W)$.

From this point of view, there is an isomorphism between the new descent algebra $\sum_{W}\left(A_{n}\right)$ and $G\left(W\left(A_{n}\right)\right)$. This isomorphism is stated as follows:
Proposition 2.12 The linear map $\psi$ defined by the images $\psi\left(\left[x_{J}\right]\right)=\chi_{J}$ is an isomorphism from the new descent algebra $\sum_{W}\left(A_{n}\right)$ to the $G\left(W\left(A_{n}\right)\right)$.

Proof. The linear map $\psi$ is a composition of $\Theta$ and $\varphi$ defined in Proposition 2.11 and 2.10, respectively. This completes the proof.

Thus, we may identify the new descent algebra with this ring of class functions.

\section{References}

[1] R. W. Carter, Simple Groups of Lie Type, John Wiley and Sons, London, 1989.

[2] L. Solomon, A Mackey Formula in the Group Ring of a Coxeter Group, Journal of Algebra 41, (1976), 225-264.

[3] F. Bergeron, N. Bergeron, R. B. Howlett and D. E. Taylor, A Decomposition of the Descent Algebra of a Finite Coxeter Group, Journal of Algebraic Combinatorics 1 (1), (1992), 23-44.

[4] M. D. Atkinson, G. Pfeiffer, S. J. Van Willigenburg, The p-Modular Descent Algebras, Algebras and Representation Theory 5, (2002), 101-113.

[5] M. D. Atkinson, Solomon's Descent Algebra Revisited, Bull. London Math. Soc. 24, (1992), 545-551.

[6] C. Bonnafe, G. Pfeiffer, Around Solomon's Descent Algebras, Algebr. Represent. Theory 11, (2008), 577-602.

[7] A. M. Garsia, C. Reutenauer, A Decomposition of Solomon's Descent Algebras, Adv. in Math. 77(2), (1989), 189-262. 